\section{Cuidados paliativos oncológicos: elementos para o debate de diretrizes nesta área}

\author{
Palliative care in cancer: \\ elements for debating the guidelines
}

Ronaldo Corrêa Ferreira da Silva 1

Virginia Alonso Hortale 2

\section{Introdução}

The aim of this study was to present elements for debating guidelines on palliative care programs for cancer. A literature survey searched various databases (MEDLINE, LILACS, and Cochrane Library), homepages of palliative care organizations, publications by renowned authors in this area, reference textbooks on the subject, relevant articles cited by these books, and the thesis/dissertation database of CAPES (the Brazilian Coordinating Body for Training University Level Personnel). The data were grouped into four thematic categories: symptomatic palliation, organization of services, $p s y$ chosocial characteristics, and spiritual characteristics. The article then proceeds to discuss cancer as a public health problem and its impact on individuals, the concept of palliative care and its context in health care, program models and their guidelines, death and dying, and care and caregivers. The study concludes by discussing the challenges for the Brazilian National Health System in structuring end-of-life programs as a consequence of the population's aging and increasing cancer incidence.

Hospice Care; Medical Care; Home Nursing; Review Literature
Hoje a ciência médica pode lutar contra uma doença potencialmente fatal e a morte, onde uma vez o médico apenas podia oferecer conforto e segurança. É comum na prática médica (ou dos profissionais de saúde) prolongar a vida a qualquer custo (e muitas vezes com sucesso). A morte, desta forma, passa a ser entendida como um fracasso e por este motivo deve ser "escondida". Nos Estados Unidos, menos de $10 \%$ da população morre devido a um infarto, acidente ou um evento inesperado. Mais de $90 \%$ morre de doença crônica, lentamente progressiva, com um período terminal de poucos meses ou semanas (como o câncer) ou de progressão lenta com períodos cíclicos de crise até advir a morte (insuficiência cardíaca e demência) 1. Das diversas causas de morte no mundo, o câncer é a única que continua a crescer independente do país ou continente e, nos países em desenvolvimento, é atualmente responsável por uma entre dez mortes 2 .

Aprender a lidar com as perdas num contexto de uma doença crônica como o câncer é um desafio que poucos se propõem a discutir, e muito menos a enfrentar. Ajudar indivíduos com doenças avançadas e potencialmente fatais (doenças terminais) e seus familiares num dos momentos mais cruciais de suas vidas é uma atividade ou um modelo de atenção à saúde que vem sendo denominado "cuidados paliativos". 
Cuidados paliativos é reconhecido como uma abordagem que melhora a qualidade de vida dos indivíduos e familiares na presença de doenças terminais. Controle dos sofrimentos físico, emocional, espiritual e social são aspectos essenciais e orientadores do cuidado. Pode ser oferecido em instituições de saúde bem como na própria residência. Pode e deve ser oferecido aos indivíduos com doença terminal (desde seu diagnóstico até o momento da morte) e aos seus familiares (durante o curso da doença e em programas de enlutamento).

Inicialmente, cuidados paliativos eram oferecidos aos indivíduos com câncer, entretanto, ao mesmo tempo em que esse tipo de cuidado ganhou prestígio, cresceu o interesse em oferecê-lo a indivíduos portadores de doenças que não o câncer. De acordo com o The Hospice Information Service do St. Christopher's Hospice, em Londres, existem mais de 7.000 hospices ou serviços de cuidados paliativos em mais de 90 países em todo o mundo. Na América Latina existem mais de 100 serviços de cuidados paliativos ${ }^{3}$. No Brasil, apesar dos dados não serem oficiais, existem cerca de 30 serviços de cuidados paliativos 4 .

Sabe-se que o câncer é reconhecido como um problema de saúde pública e que, em todo o mundo, a maioria dos indivíduos apresenta doença avançada no momento do diagnóstico 2. São também reconhecidos o impacto do câncer no indivíduo e familiares e o papel dos cuidados paliativos no controle dos sofrimentos físico, espiritual e psicossocial.

Este artigo, parte da dissertação de Mestrado de um dos autores 5 , tem como objetivo trazer alguns elementos para o estabelecimento de diretrizes para programas de cuidados paliativos no Brasil, utilizando-se do levantamento bibliográfico, da análise e da discussão das questões relacionadas à morte e ao morrer, e dos cuidados e cuidadores, fundamentais para a adequação das experiências desenvolvidas em outros países às características culturais e sociais do nosso país.

\section{Estratégia metodológica}

Foi realizado um levantamento bibliográfico, no qual foram identificados livros de referência em cuidados paliativos, bases de dados na Internet (MEDLINE, LILACS e Biblioteca Cochrane), obras de interesse citadas nas referências desses mesmos livros, textos de autores considerados fundamentais para quem trabalha com cuidados paliativos, sítios na Internet de organizações e instituições voltadas para cuidados paliativos e o banco de teses da Coordenação de Aperfeiçoamento de Pessoal de Nível Superior (CAPES).

O livro Oxford Textbook of Palliative Medicine 6 - essencial para quem trabalha com cuidados paliativos - foi considerado como referência para a discussão dos conceitos básicos em cuidados paliativos, bem como para sua historiografia.

Para a discussão dos temas morte e cuidados foram identificados, principalmente, autores nacionais. Esta estratégia teve como objetivo aproximar esta discussão ao contexto cultural nacional.

Nos sítios da Internet de instituições nacionais e internacionais que lidam com cuidados paliativos foram encontradas informações como: estrutura e funcionamento das mesmas, produtos e serviços oferecidos, informações técnicas (artigos científicos), sugestões de leitura, calendário de atividades e links para endereços de outros sítios. A seleção das instituições teve como critérios: pioneirismo, representatividade nacional e internacional, serem financiadoras de programas e projetos em cuidados paliativos, experiência em ensino e pesquisa em cuidados paliativos, e serem consideradas instituições de excelência.

O levantamento nas bases de dados foi realizado entre agosto e outubro de 2003. Para a consulta nas bases de dados MEDLINE, LILACS e Biblioteca Cochrane, foi utilizado o portal da Biblioteca Virtual em Saúde - BVS (http:/ / www. bvs.br). Na base MEDLINE (1993-2003), optouse pela revisão dos últimos seis anos, devido ao fato dos livros de referência em cuidados paliativos terem suas últimas edições publicadas no final da década de 90, conseqüentemente, utilizando referências anteriores à data de publicação. Nessa base de dados foi utilizada a pesquisa estruturada por meio do formulário básico. Como descritor de assunto usou-se o termo “cuidados a doentes terminais", selecionando o operador lógico "and" para o tipo de publicação artigo de revista e somente nos idiomas inglês e português. Foram selecionados para análise somente os artigos publicados entre 1998-2003, num total de 672 artigos.

Na base LILACS, optou-se pela revisão da literatura sem restrição de período e procedeuse a uma pesquisa estruturada utilizando o formulário básico, tendo como descritor de assunto os termos "cuidados paliativos", "tratamento paliativo" e "assistência paliativa”. Foi selecionado o operador lógico "and" para o tipo de publicação artigo de revista e restringiu-se a busca aos idiomas inglês, português e espanhol. Embora não se tenha estabelecido restrições de período, os artigos mais antigos datavam de 
1991. Foram encontrados um total de 78 artigos, sendo que 33 se repetiam.

$\mathrm{Na}$ base da Biblioteca Cochrane foram realizados dois modos de busca, com e sem restrição de período. No primeiro, selecionando o grupo "pain, palliative care e supportive care" na opção tópicos, e no segundo, realizando uma pesquisa (de 1990 a 2003) refinada sem restrições, utilizando a expressão "palliative". No primeiro modo de busca foram identificadas 16 publicações, enquanto no segundo foram identificadas 1.359. Do segundo modo de busca, selecionou-se para análise as publicações sobre revisões sistemáticas (145), revisões sobre efetividade (48), avaliações tecnológicas em saúde (23) e avaliação econômica do NHS (147).

O banco de teses da CAPES (http://www. capes.gov.br) foi utilizado de forma complementar para coletar teses e dissertações do período de 1991 até 2002 referentes aos temas "câncer", "cuidados paliativos", "cuidados ao final da vida", "medicina paliativa", "morte", " finitude" e "cuidados". Foram encontradas 11 teses/dissertações.

O objetivo do levantamento foi destacar publicações recentes que acrescentassem novas discussões à luz dos textos e de autores de referência, e identificar as tendências da produção científica na área, de acordo com as categorias temáticas.

Os trabalhos foram agrupados em quatro categorias temáticas: controle de sintomas, organização de serviços, aspectos psicossociais e espirituais. Na primeira categoria (controle de sintomas) foram selecionados trabalhos sobre controle da dor e outros sintomas, atenção à fase terminal da doença e aspectos éticos das intervenções. Na segunda categoria (organização de serviços) foram selecionados trabalhos que definiam o papel da equipe interdisciplinar, as experiências particulares de cada instituição, as discussões sobre modelos de atenção, o papel da educação e formação de recursos humanos, as iniciativas de controle da qualidade de serviços e pesquisa em cuidados paliativos. Na terceira categoria (aspectos psicossociais) foram selecionados trabalhos que discutiam o impacto da doença terminal nos indivíduos e familiares, os transtornos psicológicos mais freqüentes, os programas de apoio psicossocial no período da doença e após a morte, e os transtornos psicológicos na equipe de saúde. Na quarta categoria (aspectos espirituais) foram selecionados trabalhos que discutiam o papel do apoio espiritual nos cuidados ao indivíduo em fase terminal da doença.

Na base MEDLINE, observou-se o predomínio de artigos na categoria organização de ser- viços (562), seguido da categoria controle de sintomas (83) e um número bastante reduzido de artigos nas categorias aspectos psicossociais (15) e aspectos espirituais (12). Entendeu-se que essa distribuição deveu-se ao fato dos cuidados paliativos nos países desenvolvidos ocorrerem um grande número de pesquisas voltadas para o desenvolvimento organizacional visando à melhoria da qualidade dos serviços. Identificou-se também um pequeno número de publicações relativas aos aspectos espirituais, apesar deste número estar aumentando nos últimos anos.

$\mathrm{Na}$ base LILACS, que registra as pesquisas produzidas na América Latina e Caribe, o predomínio foi de publicações na categoria de controle de sintomas (35) e organização de serviços (9), de um total de 45 artigos (78 artigos no total menos 33 que se repetiam). Esse achado reforça a afirmação de Singer 7 de que os países em desenvolvimento ainda estão em fase de construção de modelos e programas de cuidados paliativos, uma vez que a preocupação ainda é com a adoção de práticas de controle de sintomas e organização de serviços.

Na base da Biblioteca Cochrane, das revisões sistemáticas completas (74 em 145), a maioria se relacionava com a categoria controle de sintomas (63). Das revisões sobre efetividade, avaliação tecnológica em saúde e avaliação econômica do NHS, a maioria se relacionava com a categoria controle de sintomas (39, 17 e 90, respectivamente).

No banco da CAPES foi identificado um número reduzido de teses/dissertações. Apenas 11 abordavam diretamente o tema, sem que qualquer uma delas salientasse as questões conceituais e de organização de programas e serviços.

A análise de todo o material bibliográfico (livros de referência, bases de dados, sítios na Internet, textos de autores referenciais e banco de teses e dissertações) foi realizada em três fases: crítica do material coletado, análise e interpretação dos textos. É importante ressaltar que a revisão da literatura serviu como um balizador para a construção do texto, ou seja, o predomínio de publicações científicas na categoria controle de sintomas (35) e organização de serviços (9) nos países em desenvolvimento (base de dados LILACS) indicava a necessidade do aprofundamento da discussão sobre organização de serviços. O resultado é apresentado a seguir com a seguinte disposição: câncer como um problema de saúde pública; câncer e seu impacto no indivíduo; cuidados paliativos e alívio da dor; modelos de programas e suas diretrizes; a morte e o morrer; cuidados e cuidadores. 


\section{O câncer como um problema de saúde pública}

Segundo a Organização Mundial da Saúde (OMS), ocorre anualmente em todo o mundo mais de 10 milhões de casos novos e 6 milhões de mortes por câncer. Cerca de 4,7 milhões de casos novos ocorrem nos países economicamente desenvolvidos, enquanto 5,5 milhões ocorrem nos países economicamente menos desenvolvidos. Nos países desenvolvidos o câncer se apresenta como a segunda causa de morte por doença, enquanto que nos países em desenvolvimento como terceira causa nesta categoria 2 .

Atualmente o câncer é responsável por $12 \%$ de todas as mortes no mundo. Em aproximadamente vinte anos, o número de mortes anuais por câncer irá quase que duplicar, com mais de $60 \%$ dos casos novos e mortes ocorrendo nos países menos desenvolvidos economicamente. Em todo o mundo, a maioria dos indivíduos diagnosticados com câncer apresenta doença avançada e incurável no momento do diagnóstico 2. Das 56 milhões de mortes que ocorrem anualmente no mundo, $85 \%$ são nos países menos desenvolvidos economicamente. Cerca de $10 \%$ destas mortes são devidas ao câncer ${ }^{2}$. Apesar desses dados apontarem para uma maior necessidade de cuidados paliativos nos países economicamente menos desenvolvidos, a maioria das pesquisas e das iniciativas em cuidados paliativos tem ocorrido nos países economicamente desenvolvidos. Essa situação pode se caracterizar como um paradoxo, uma vez que a aplicação acrítica de estratégias e conceitos dos países economicamente mais desenvolvidos nos países menos desenvolvidos leva a distorções e inadequações organizacionais 7 .

Governos, sociedades médicas e de enfermeiros e organizações não-governamentais reconhecem a importância dos cuidados paliativos e reafirmam a integração dos seus princípios aos programas de saúde pública, principalmente os Programas Nacionais de Controle de Câncer (como também AIDS). Todos os países deveriam implementar programas de cuidados paliativos com a finalidade de melhorar a qualidade de vida da maioria dos indivíduos com câncer (e outras doenças potencialmente fatais) e seus familiares 2 . Esses programas estariam em condições de oferecer serviços de controle da dor (e outros sintomas), suporte social, espiritual e psicológico. No entanto, sua organização é complexa pois depende da disponibilidade de recursos das mais variadas ordens. Se os recursos são escassos, seria aconselhável garantir que padrões mínimos de controle da dor e cuidados paliativos fossem incor- porados progressivamente em todos os níveis de atenção e que se alcançasse uma grande cobertura de indivíduos por meio de atendimento domiciliar 2.

Das doenças crônicas degenerativas, o câncer é uma das que mais trazem transtornos aos indivíduos e seus familiares. Sofrimentos de diversas dimensões acometem tanto os portadores da doença como seus familiares e cuidadores. Entender o impacto do câncer nos indivíduos é essencial para estabelecer estratégias de cuidados.

\section{O câncer e seu impacto no indivíduo}

Além da dor e outros desconfortos físicos para o indivíduo, o câncer causa impacto quer de ordem psíquica, social e econômica, tanto para ele como para seus familiares. Nos países subdesenvolvidos, as perdas são mais intensas, principalmente se não existir um sistema formal de saúde em condições de atender às suas necessidades. Perdas econômicas devidas ao câncer são óbvias: custos diretos (hospitalares, medicamentos, outros serviços de saúde) e custos indiretos (potencial produtivo perdido e sobrecarga do sistema previdenciário) 2 .

Um estudo publicado em 1996 estimou os custos diretos nos cuidados com o câncer nos Estados Unidos em 27,5 bilhões de dólares, e os custos indiretos com a mortalidade prematura em torno de 59 bilhões de dólares 2 . Estima-se que nas primeiras décadas do século XXI, grande parte dos recursos estará concentrado nos cuidados ao final da vida. Em todo o mundo mais indivíduos estarão morrendo de doenças crônicas ou progressivas do que de condições agudas 2. Nos Estados Unidos, os gastos com cuidados ao final da vida representam cerca de $27 \%$ do orçamento do Medicare, equivalendo a $10-12 \%$ do total de gastos em saúde com este tipo de seguro 8 .

Independente do prognóstico, o diagnóstico inicial de câncer é ainda entendido por muitos indivíduos como um evento que põe suas vidas em risco 2 . Transtornos de ordem psíquica são freqüentes e levam a uma diminuição da sua qualidade de vida e de seus familiares. Segundo relatório da OMS de 2002, cerca de um terço dos indivíduos com câncer apresenta algum grau de ansiedade e/ou depressão ${ }^{2}$. Muitos profissionais de saúde acreditam ser natural estar deprimido quando se está morrendo. Entretanto, é importante saber que esta complicação é tratável. Os profissionais de saúde necessitam estar atentos para identificá-la e capacitados para instituir o melhor tratamento 
para cada caso. Outra forma comum de sofrimento psíquico (emocional) é a ansiedade. Ela é capaz de exacerbar outras formas de sofrimento, como por exemplo a dor, e também é tratável. No cotidiano dos cuidados paliativos, percebe-se que o sofrimento psíquico é tão perturbador quanto o sofrimento físico, e para muitos é menos tolerável do que o sofrimento físico. Nem os profissionais de saúde vinculados à assistência estão imunes aos efeitos dos cuidados aos indivíduos com câncer 2. É comum os profissionais de saúde que lidam com indivíduos muito enfermos ou moribundos apresentarem exaustão emocional, insônia, absenteísmo, abuso de álcool e outras substâncias, além de sintomas somáticos 2 .

As relações sociais costumam se modificar pela presença do câncer. Tanto para aqueles com câncer quanto para seus familiares e amigos, questões como perda do poder aquisitivo, isolamento social, tensão familiar, manutenção dos laços de amizade, capacidade de manter o emprego ou os estudos, entre outros, são comuns e desafiadores para os que convivem com o câncer. Muitas vezes, um diagnóstico de câncer implica não somente em uma diminuição no salário, mas uma perda das reservas econômicas na busca por tratamentos que nem sempre aumentam a sobrevida. Atitudes sociais perante a doença podem isolar uma pessoa, e os cuidados prestados podem levar a situações estressantes na relação íntima com o enfermo. Medo e luto antecipado podem ocorrer nos indivíduos com câncer terminal e seus cuidadores, podendo causar mudanças na orientação das relações afetivas.

Os textos literários são excelentes fontes para se obter relatos da experiência do morrer e do enlutamento antecipado. Um dos textos considerados mais ilustrativos desta experiência é o conto A Morte de Ivan Ilitch, de Leon Tolstoi 9. Nele, Tolstoi relata a trajetória de um funcionário público na Rússia czarista nos últimos meses de sua vida. O autor reflete sobre a condição do personagem como portador de uma doença intratável e fatal. Descrevendo as diversas fases de seu sofrimento (do diagnóstico à morte), apresenta questões como a relação médico-paciente, a autonomia do indivíduo, as relações familiares, o isolamento e a perda da capacidade de trabalho, entre outras.

Portanto, ao se planejar ações para controlar os impactos do câncer nos indivíduos e seus familiares, deveria ter-se a sensibilidade (e a capacidade) de identificar desordens do campo físico, bem como do campo psíquico, social e espiritual. Foi com o objetivo de identificar e cuidar destas diversas dimensões do sofrimen- to humano que surgiu a modalidade de cuidado chamada cuidados paliativos.

\section{Cuidados paliativos e alívio da dor}

Na primeira definição da OMS para cuidados paliativos, em 1998, estes foram categorizados como o último estágio de cuidado: "cuidados oferecidos por uma equipe interdisciplinar voltados para pacientes com doença em fase avançada, ativa, em progressão, cujo prognóstico é reservado e o foco da atenção é a qualidade de vida" 6 (p. 3). Entretanto, é sabido que os cuidados paliativos podem e devem ser oferecidos o mais cedo possível no curso de qualquer doença crônica potencialmente fatal, para que esta não se torne difícil de tratar nos últimos dias de vida 2 . A mais recente definição da OMS estabelece que "cuidados paliativos é uma abordagem que melhora a qualidade de vida dos pacientes e seus familiares frente a problemas associados à doença terminal, através da prevenção e alivio do sofrimento, identificando, avaliando e tratando a dor e outros problemas, físicos, psicossociais e espirituais" 2 (p. 84).

Além da dor (um dos sintomas mais freqüentes), outros sintomas acometem os indivíduos com câncer, como: anorexia, depressão, ansiedade, constipação, disfagia, dispnéia, fraqueza, entre outros. Todos diminuem de algum modo sua qualidade de vida, merecendo, portanto, a atenção dos profissionais de saúde. À medida que a doença progride, maior é a necessidade de cuidados paliativos, o que os torna quase que exclusivos ao final da vida, porém, não terminando com a morte do indivíduo com câncer. Segundo a OMS 2, a proporção de indivíduos com câncer que requerem cuidados paliativos nos países menos desenvolvidos economicamente é de $80 \%$. Como o sofrimento de um indivíduo pode se apresentar sob diversas dimensões (físico, psicológico, espiritual, social, econômico), assim também devem ser as dimensões do cuidado aos indivíduos sob cuidados paliativos.

Atualmente, vêm sendo empregados com mais freqüência e indistintamente os termos cuidados paliativos, medicina paliativa, hospi$c e$ (sem tradução para o português), nursing ou residential homes (asilos ou casas de repouso). É preciso conceituá-los e estabelecer as diferenças. A definição de cuidados paliativos da OMS já foi apresentada. Hospice pode ser utilizado para descrever uma filosofia de trabalho voltada aos indivíduos com doenças terminais (Estados Unidos), um prédio ou edifício onde são praticados os cuidados paliativos (Estados 
Unidos, Inglaterra) ou o programa de cuidados regularmente oferecido ao final da vida (Estados Unidos, Inglaterra). Medicina Paliativa é uma nova especialidade médica reconhecida na Inglaterra em 1987 e, em muitos países, uma área de especialização oferecida por programas de pós-graduação. Nursing homes ou residential homes poderiam ser considerados o equivalente aos asilos ou casa para idosos no Brasil. Não possuem uma equipe especializada em cuidados paliativos, sua clientela permanece mais tempo internada, o número de indivíduos com câncer é pequeno e normalmente é constituída de solteiros ou viúvos 10 . Independente da confusão acerca dos conceitos, os cuidados paliativos desenvolveram-se como uma resposta às contínuas dificuldades em cuidar dos indivíduos com câncer e suas famílias. A importância em entender esse indivíduo e sua família como uma unidade é uma característica desse cuidado.

A discussão atual volta-se para a possibilidade de entender cuidados paliativos como um conceito que permeia todo o cuidado, ou seja, do diagnóstico à morte, incluindo o processo de luto. Em síntese, cuidados paliativos e hospice afirmam seis princípios 1 : valoriza a vida $\mathrm{e}$ considera a morte como um processo natural; nem abrevia nem prolonga a vida; provê o alívio da dor e outros sintomas; integra os aspectos psicológicos e espirituais dos cuidados, permitindo oportunidades para o crescimento; oferece uma equipe interdisciplinar e um sistema de suporte para a família durante a doença do indivíduo e no período de enlutamento. Utilizando estes princípios como norteadores das ações, passamos, a seguir, à análise dos modelos de programas.

\section{Modelos de programas e suas diretrizes}

Como já apresentado anteriormente, cuidados paliativos se propõem a aliviar o sofrimento e melhorar a qualidade de vida dos indivíduos com doença avançada e seus familiares por meio de um conhecimento específico e habilidades, incluindo boa comunicação com estes indivíduos e seus familiares; controle da dor e outros sintomas; suporte espiritual, psicossocial e no enlutamento; e coordenação dos diversos serviços médicos e sociais 11 .

Geralmente os cuidados paliativos são oferecidos por meio de programas inseridos ou não em instituições hospitalares. Nos programas vinculados a instituições hospitalares, torna-se claro o esforço individual de alguns pro- fissionais, considerados inovadores por perceberem a necessidade de oferecer cuidados paliativos em suas unidades de saúde. Recentemente, nos Estados Unidos, os cuidados paliativos foram reconhecidos como um componente essencial nos cuidados do câncer. Embora o número de programas em cuidados paliativos tenha crescido, o número de programas inseridos em instituições hospitalares ainda é pequeno 12.

Quando não inseridos em uma instituição hospitalar, os programas de cuidados paliativos podem estar inseridos em asilos, casas de repouso, moradias assistidas, hospices, clínicas e no domicílio (home care). Iniciativas para implantar e manter programas de cuidados paliativos deveriam estar adequadas às necessidades quer dos usuários, dos provedores, dos serviços de saúde, quer da comunidade. Modelos de programas para adultos não são considerados adequados para atender às necessidades de crianças e adolescentes 13.

O maior desafio dos cuidados paliativos é integrar-se aos cuidados curativos. Paliar é uma dimensão crítica dos cuidados em saúde e todos os profissionais de saúde deveriam saber quando os cuidados paliativos são necessários. Quando qualquer indivíduo se aproxima dos últimos momentos de uma condição de saúde debilitante, a necessidade de cuidados paliativos aumenta. Neste momento (e após o óbito), assegurar este tipo de atenção propicia um cuidado de qualidade não importando se oferecido em uma instituição de saúde ou na residência do indivíduo.

Os modelos de programas e sua inserção no sistema de saúde podem se dar das seguintes formas 13: (1) consultoria - consiste de avaliações de médico, enfermeiro e/ou assistente social, geralmente em um hospital, clínica, hospice, asilo ou em domicílio (home care); (2) unidade para pacientes internos - geralmente localizada num hospital, hospice ou asilo; (3) consultoria e unidade para pacientes internos geralmente localizado em um hospital, hospice ou asilo; (4) unidade para pacientes externos e consultoria - geralmente localizada em um hospital, hospice ou clínica (com/sem home care); (5) unidade para pacientes externos e internos e consultoria - geralmente localizado em um hospital ou hospice (com/sem home care).

É importante salientar que não existe um modelo ideal. A escolha de um ou mais modelos é definida pelas necessidades da população, dos provedores (públicos ou privados) e dos serviços de saúde. Os programas de cuidados paliativos, independente de estarem inseridos em unidades hospitalares ou não, deveriam seguir certas diretrizes que possibilitariam 
alcançar os objetivos de aliviar o sofrimento e melhorar a qualidade de vida dos indivíduos com doença avançada e seus familiares. Estas diretrizes podem ser agrupadas em seis domínios: físico, psicológico, social, espiritual, cultural e estrutural 14.

- Domínio físico: avaliação interdisciplinar do indivíduo e sua família; cuidados centrados nele e em sua família (respeito aos seus desejos); controle dos sintomas baseado em evidências; adequação do ambiente onde o cuidado é realizado.

- Domínio psicológico: avaliar o impacto da doença terminal no indivíduo e familiares; estabelecer um programa de enlutamento; estabelecer um programa de cuidados do staff clínico.

- Domínio social: avaliar as necessidades sociais do indivíduo e sua família; estabelecer uma abordagem individualizada e integrada; manter a comunidade alerta sobre a importância dos cuidados paliativos; incluir a proposta de cuidados paliativos na formulação de políticas sociais e de saúde.

- Domínio espiritual: as crenças religiosas devem ser reconhecidas e respeitadas; suporte espiritual e religioso para indivíduos e familiares. - Domínio cultural: o serviço de cuidados paliativos deve atender às necessidades culturais dos indivíduos e familiares; deve refletir a diversidade cultural da comunidade a qual serve; programas educacionais devem ser oferecidos aos profissionais para que atendam a esta diversidade cultural.

- Domínio estrutural: equipe interdisciplinar (serviços médicos, de enfermagem e auxiliares); incorporar voluntários; treinamento especializado em cuidados paliativos para a equipe interdisciplinar; incorporar atividades de melhoria da qualidade dos serviços e de pesquisa clínica e de processos gerenciais.

O National Consensus Project for Quality Palliative Care 13 acrescenta mais duas dimensões (ou domínios): domínio dos cuidados ao moribundo (últimas 24-48h) e domínio dos aspectos éticos e legais do cuidado (bioética do final da vida). Os objetivos das diretrizes seriam promover a qualidade dos serviços; reduzir as variações entre serviços novos e os já existentes; estimular a continuidade dos cuidados em todos os níveis de atenção; facilitar parcerias entre os programas, a comunidade e outras unidades de atenção à saúde.

Os cuidados paliativos são oferecidos, predominantemente, para indivíduos ao final da vida (moribundos). Da compreensão das crenças e costumes em relação à morte e ao morrer, emergirão propostas para intervenções ao final da vida coerentes com a cultura local.

\section{A morte e o morrer}

A sociedade contemporânea tem cada vez mais dificuldade em pensar a questão da morte e do morrer, apesar de tornar-se uma sociedade de risco, com múltiplas possibilidades de experimentar a vulnerabilidade e enfrentar a morte em situações violentas de vários tipos. No século XX, a morte outrora tão presente na vida familiar, vai sendo recalcada. A presença da morte gera incômodos provocados pela sua presença numa sociedade que vive pelo prazer. A partir da década de 30, o local da morte é transferido das residências para os hospitais. A expulsão da morte (individual) da cena contemporânea, da interdição do luto e do direito de chorar seus mortos, são componentes característicos da civilização contemporânea ocidental. Este recalcamento do pensamento da morte, na persistência das ocasiões de experiência da morte, pode ser considerado como uma conseqüência do processo civilizador 15.

Uma vez que a morte é ocultada, e que sobre ela se fixem um conjunto de valores negativos, o moribundo é algo que incomoda. Seu sentido no ambiente hospitalar é negativo, é algo que precisa ser escondido sob o discurso técnico da biomedicina, irreconhecível como humano. Ao mesmo tempo, na sociedade avançada, a consciência da legitimidade moral da cultura do respeito da autonomia do indivíduo acrescenta uma nova atmosfera ao redor do morrer, na qual a tarefa dos médicos de fazer todo o possível para impedir ou postergar a morte é discutível 15. É sobre essa construção social que os cuidados paliativos emergem como uma alternativa às práticas instituídas. Cuidados paliativos ocupam o espaço entre a competência técnica da medicina curativa e a cultura do respeito à autonomia do indivíduo no que se refere às suas decisões extremas (incluindo a prerrogativa de dizer que não quer mais viver sofrendo) 15.

Toda sociedade tem de dar conta da morte e dos mortos, e, por conta disso, há padrões dicotômicos de comportamento 16. De um lado há sistemas que se preocupam com a morte, de outro, os que se preocupam com o morto. Há uma tendência em ver a morte como importante, descartando o morto, e uma outra que tende a ver o morto como básico, descartando a morte. Na sociedade moderna não há luto nem qualquer tipo de contato com os mortos. Uma atitude inversa é encontrada nas culturas tribais e tradicionais (relacionais), onde o sujeito social não é o indivíduo, mas as relações entre indivíduos. Nelas, ocorre uma grande elaboração relativa ao mundo dos mortos, que são in- 
vocados, chorados, relembrados, homenageados e usados sem cerimônia pela sociedade. Tudo isso acompanhado de um silêncio profundo sobre a morte 16 .

No Brasil, segundo Da Matta 16, se fala muito mais dos mortos do que da morte. Isso implica uma contradição, uma vez que falar dos mortos já é uma forma sutil e disfarçada de negar a morte, fazendo prolongar a memória do morto. Para esse autor, a morte no Brasil é concebida como uma passagem de um mundo a outro, uma metáfora de subida ou descida, algo verticalizado como a própria sociedade brasileira. Finaliza suas considerações com uma frase primorosa: “... podemos entender por que no Brasil a morte mata, mas os mortos não morrem" 16 (p. 158).

Dentre os que já tiveram a oportunidade de acompanhar as fases finais de um indivíduo ao final da vida, muitos experimentaram desconforto, principalmente se esse indivíduo foi um ente querido. É fácil compreender a dificuldade que os médicos, bem como outros profissionais de saúde, têm em lidar com essas situações. Muitas vezes, sob o disfarce da afirmativa "não há mais nada a fazer por ele", esconde-se a dificuldade em lidar com a morte. Na verdade, não é exatamente a morte a principal dificuldade, mas sim o acompanhamento do indivíduo que está morrendo. Nas últimas décadas, a literatura tem sido pródiga em publicações sobre as atitudes do médico diante da morte e do doente terminal 17. Apesar da grande projeção sobre o assunto em anos recentes, os sentimentos dos médicos e dos estudantes de medicina em relação à morte e o morrer são pouco conhecidos.

É importante destacar que os grandes progressos da medicina instrumental não foram acompanhados de progressos na atenção aos indivíduos com câncer. É como se os progressos científicos, voltados para a efetividade, fossem incompatíveis com os cuidados, voltados para a afetividade. Os hospitais dispõem de equipamentos e tecnologias de última geração, mas são incapazes de desempenhar sua função primeira: oferecer hospitalidade. "Ao secularizar os tratamentos, o hospital parece que se desumanizou" 18 (p. 43). Daí surgirem os movimentos para humanização dos serviços, como se a aplicação da técnica fosse "desumana". O fator humano parece formado para desempenhar papel de máquina reparando outras máquinas. Não são formados para desempenhar uma parte significante de sua função que é cuidar de seres humanos.

Diversos atores sociais estão envolvidos nos cuidados ao moribundo e desempenham papel importante no compromisso de oferecer qualidade de vida e suporte aos mesmos. Não somente os profissionais de saúde, mas também familiares e amigos, comunidade e instituições sociais, deveriam ser motivados para os cuidados ao indivíduo com câncer e, em conseqüência, serem qualificados para tal.

\section{Cuidados e cuidadores}

Com o progresso da medicina, principalmente no último século, os cuidados ao final da vida passaram a ser realizados, sobretudo nos países desenvolvidos, em um ambiente impessoal, cercado de pessoas estranhas, muitas vezes sob monitoramento de aparelhos eletrônicos e submetidos a procedimentos médicos invasivos. Esse panorama reflete o que é denominado "medicalização da morte" 19. Na contramão desse processo encontram-se os cuidados paliativos ou os cuidados ao final da vida. O cuidado, segundo Boff 20 , possui muitas repercussões, conceitos afins que emergem do cuidado e o traduzem em atitudes e ações. Uma dessas repercussões é a compaixão. Trata-se da capacidade do ser humano de compartilhar o sofrimento (ou alegrias) do outro, construindo a vida em sinergia com este. Não é passiva e nem representa um sentimento de piedade. Como salienta Caponi 21, a motivação para a compaixão com a pessoa que sofre pode ser resultado de um sentimento de que algo semelhante pode acontecer a qualquer um (solidariedade genuína), de outra forma, pode também ser motivada por um sentimento de piedade pelos que sofrem ou pelo simples fato de pensar que socorrer os infortunados contribui para o bem comum. A solidariedade genuína procura tomar como ponto de partida as diferenças entre os que sofrem. Existindo a diferença, o diálogo é essencial para se criar vínculos. E nesse diálogo são estabelecidos modelos de atenção que respeitam as crenças e saberes daqueles indivíduos, e principalmente não permitem a exclusão ou a adoção de projetos (de cuidados) préestabelecidos.

Em geral, a maior parte do tempo do último ano da vida dos indivíduos é passada em casa, muito embora $90 \%$ deles passem algum tempo em um hospital e 55\% das mortes ocorram neste local 22. As obrigações e recompensas de cuidar de pessoas no seu último ano de vida são compartilhadas pelos cuidadores informais (parentes, amigos e vizinhos) e formais (profissionais de saúde) 22 . Cerca de $75 \%$ das pessoas recebem cuidados em casa por meio de cuidadores informais, sendo que a maioria deles é de mulheres. Quase a metade dos indivíduos com 
câncer recebe cuidados de dois ou mais parentes, freqüentemente a esposa e um filho adulto. Aproximadamente $2 / 3$ destes indivíduos recebem alguma forma de cuidado formal 22.

Com a transição demográfica observada no Brasil, uma significativa parcela dos indivíduos com câncer está em idade avançada. Essa população (bem como a idosa) é freqüentemente dependente de uma ou mais pessoas. A atual tendência em muitos países, e no Brasil também, é sugerir a permanência dessa população em suas casas, sob os cuidados de sua família 23 . Em vários programas de cuidados paliativos no Brasil e no mundo são utilizados cuidados domiciliares como estratégia de oferecer cuidados humanizados.

A delicada relação entre o cuidador e o indivíduo ao final da vida permite inferir que a humanização, muitas vezes, está longe de acontecer. "Não é necessariamente o local que humaniza a relação terapêutica, mas, sim, o interesse empático que o profissional desenvolve pelo paciente" 24 (p. 991). Se o objetivo é aperfeiçoar a qualidade dos cuidados oferecidos a essa população, torna-se fundamental reconhecer as necessidades dos cuidadores formais $\mathrm{e}$ informais e estabelecer estratégias de suporte para os mesmos.

Vários profissionais de saúde (cuidadores formais) estão envolvidos com os cuidados ao indivíduo no final da vida e em diferentes locais (hospitais, ambulatórios, asilos e residências). As atividades assistenciais levam a diversas formas de estresse, mas também proporcionam momentos de satisfação. É reconhecida a ocorrência da Síndrome de Burnout (Síndrome de Esgotamento Emocional) entre os profissionais de saúde que lidam com a morte ou situações próximas da morte 25. É importante ressaltar que a prevalência de morbidade psiquiátrica entre médicos e enfermeiras que trabalham com cuidados paliativos e hospices é menor do que em outros profissionais de saúde 26 .

Manter e aprimorar a saúde mental dos trabalhadores é essencial para os próprios profissionais de saúde, bem como para a qualidade dos cuidados oferecidos aos indivíduos. Algumas estratégias sugeridas para melhorar a saúde mental dos profissionais de saúde em cuidados paliativos são: manter uma cultura de cuidados paliativos, oferecer treinamento em comunicação e gerenciamento, oferecer supervisão clínica adequada e que inclua as diversas dimensões dos cuidados ao indivíduo (física, social, psicológica e espiritual) e oferecer um serviço de saúde mental independente que atenda problemas de caráter pessoal e os relacionados ao trabalho 22 .
Sem o apoio da família e dos amigos (cuidadores informais), seria difícil para muitos indivíduos permanecerem em casa. Normalmente os familiares assumem os cuidados, no entanto amigos e vizinhos também podem assumir papéis de cuidadores informais. A tarefa de cuidar está sob a responsabilidade quase exclusiva da família, uma vez que a organização comunitária também se mostra bastante deficiente 26 . Os cuidadores informais necessitam de informação e orientação sobre a doença do indivíduo e como cuidá-lo, além de suporte psicológico e social 22.

Estar bem informado sobre a doença do indivíduo parece diminuir a ansiedade referente a medos improváveis e irreais. Como a grande maioria dos cuidadores informais não possui treinamento em enfermagem, beneficiam-se de instruções práticas, tais como administrar remédios, mudança de posição no leito, entre outros. É alta a prevalência de sintomas como ansiedade e depressão, como também distúrbios do sono e perda de peso 22 . A preferência do cuidador pelo local da morte do indivíduo é uma informação que freqüentemente não está disponível. Desgaste psicológico, falta de apoio de enfermagem e ausência de equipamentos são as justificativas mais comuns para admitir no hospital indivíduos moribundos 22.

Quanto aos cuidados realizados por um cuidador informal, dois aspectos devem ser salientados: as condições necessárias à manutenção dos cuidados na residência e as condições que realmente a família dispõe para ser responsabilizada pelo mesmo. Sabe-se que os gastos familiares com os indivíduos ao final da vida são crescentes e, muitas vezes, o cuidador também é o provedor (ou um dos provedores) dos recursos financeiros, e que, infelizmente, o sistema de seguridade social no Brasil não tem conseguido cumprir seu papel, recaindo sobre a família a cobertura destas falhas 26 . Outros fatores como o baixo nível de renda, baixa escolaridade, carência de serviços públicos e de saneamento básico são indicadores da precariedade das condições e qualidade de vida, e apontam para dificuldades no cuidado desses indivíduos na comunidade 26 .

\section{Considerações finais}

O objetivo maior deste trabalho foi apresentar a discussão atual sobre os cuidados paliativos oncológicos. Para isso, a estratégia foi mostrar um panorama amplo sobre cuidados paliativos no que diz respeito a conceitos, organização de programas e desafios futuros. 
Entende-se que essa discussão é essencial para a definição de políticas, programas e serviços em cuidados paliativos. Procurou-se deixar claro que o período que antecede a morte de um ser humano portador de uma doença crônica avançada, progressiva e incurável é talvez um dos períodos mais importantes de sua vida. Não somente pelo seu caráter irremediável, mas principalmente pelos distúrbios psíquicos, financeiros, afetivos e físicos que comprometem os indivíduos, seus familiares e cuidadores. O modo como os indivíduos morrem modificou-se nos últimos cinqüenta anos. Passou-se a "medicalizar" ou "institucionalizar" a morte. Este é o final do processo iniciado quando a ciência assumiu o compromisso de lutar contra a morte. Como conseqüência, a morte significa derrota, vergonha e falha; tudo que o sistema moderno de saúde precisa esconder sob suas instituições.

Na década de 60, inicialmente na Inglaterra e posteriormente estendendo-se ao restante da Europa e aos Estados Unidos, a idéia de uma morte mais digna, menos sofrida, próxima das pessoas que se ama e que nos são caras, proporcionou a criação de um movimento de cuidados mais humanizado, integral, voltado mais especificamente para indivíduos com doenças crônicas, progressivas e sem possibilidade de tratamento curativo 5 . Foi a partir desse movimento que as discussões sobre os cuidados aos indivíduos ao final da vida passaram a ganhar espaço nos serviços de saúde. Se for admitido que a natureza da formação do profissional de saúde que lida com a terminalidade é complexa, será fácil compreender porque a preocupação com a formação de recursos humanos neste campo é mais do que urgente, porque passa a exigir atenção a todas as dimensões do ser (plano físico, espiritual, social, psicológico e econômico).

O ser humano como tal deveria investir-se da responsabilidade e do dever de cuidar de seus semelhantes. Mas que forma de cuidado se poderia oferecer? Uma das traduções do cuidado é a compaixão. A capacidade do ser humano de compartilhar o sofrimento ou a alegria com o outro. No entanto, qual seria a motivação para a compaixão? O que motiva a compartilhar o sofrimento dos moribundos? A motivação fundamentada pela piedade ou pelo fato de que socorrendo os infortunados se estaria contribuindo para o bem comum não garante o respeito pelo beneficiado. Tornar-se-ia necessário desenvolver o sentimento de solidariedade. É na solidariedade que o diálogo se estabelece e que as crenças e saberes são respeitadas. No caso dos cuidados paliativos fica claro que características culturais, crenças e valo- res têm papel determinante na estruturação de modelos de programas e serviços.

O Brasil ainda não possui uma estrutura pública de cuidados paliativos oncológicos adequada à demanda existente, tanto sob o ponto de vista quantitativo e qualitativo. Em pior situação encontra-se o subsetor privado de saúde. Preocupante é reconhecer que a maioria dos indivíduos com câncer procura a rede pública de serviços com a doença em estágio avançado e elegíveis apenas para cuidados paliativos. Torna-se urgente o conhecimento dos conceitos fundamentais dos cuidados paliativos e hospice, bem como empreender esforços para implementar iniciativas centradas no cuidar solidário nos serviços de saúde, sejam públicos ou privados.

O sistema de saúde brasileiro enfrenta grandes desafios para o novo século. Uma das grandes questões é o aumento da população idosa e o crescente número de indivíduos com câncer e doenças cardio e cerebrovasculares. Esse cenário indica a necessidade de se estabelecerem políticas de saúde voltadas para os indivíduos ao final da vida. Por meio dos modelos de programas de cuidados paliativos e suas diretrizes de ação, se poderiam estabelecer as bases técnicas para discutir uma política nacional de cuidados ao final da vida. Reconhece-se, entretanto, que a singularidade do tema requer uma discussão multisetorial e com a participação efetiva da sociedade civil. Talvez a análise do movimento da reforma psiquiátrica brasileira possa contribuir para sugerir estratégias de ação, uma vez que observadas as devidas proporções, os dois temas (doença mental e morte) guardam semelhanças, tais como: são temas polêmicos, estigmatizados, envolvem diretamente as famílias, propõem mudanças paradigmáticas e de atitudes, envolvem mudanças na legislação, necessitam de coordenação dos diversos níveis de atenção à saúde, propiciam conflitos de interesse e acarretam ações de educação em saúde.

A contribuição deste trabalho consistiu em apresentar algumas considerações sobre cuidados paliativos oncológicos, com a finalidade de motivar um debate com a participação de todos os atores sociais interessados em promover um sistema de saúde mais justo e humanizado, e que entendam a morte como um processo natural e irremediável. Todos irão morrer um dia. Alguns, próximos aos seus entes queridos, em um ambiente familiar, com seus sintomas controlados e sua autonomia preservada. Outros, isolados, em um ambiente impessoal, com dor e outros sintomas não controlados e impossibilitados de exercer sua autonomia. A escolha depende de todos nós. 


\section{Resumo}

Este artigo tem como objetivo apresentar elementos para o debate de diretrizes em programas de cuidados paliativos no Brasil. Foi construído por meio de levantamento bibliográfico em base de dados (MEDLINE, LILACS e Biblioteca Cochrane), em sítios na Internet de organizações e instituições interessadas em cuidados paliativos, em textos de autores fundamentais na área, em livros de referência, em obras citadas nas referências destes mesmos livros e no Banco de Teses da CAPES. Os dados foram agrupados em quatro categorias temáticas: controle de sintomas, organização de serviços, aspectos psicossociais e espirituais. Com isso, procedeu-se a discussão do câncer como um problema de saúde pública e seu impacto no indivíduo, o conceito de cuidados paliativos e seu contexto na atenção à saúde, os modelos de programas e suas diretrizes, a morte e o morrer e os cuidados e cuidadores. O artigo termina apontando os desafios do sistema de saúde brasileiro em estruturar programas de cuidados ao final da vida em conseqüência do envelhecimento da população e do aumento da incidência do câncer.

Cuidados Paliativos; Cuidados Médicos; Assistência Domiciliar; Literatura de Revisão

\section{Colaboradores}

R. C. F. Silva contribuiu para concepção e desenho do estudo; coleta, análise e interpretação dos dados; redação do artigo para submissão à publicação. V. A. Hortale contribuiu com a revisão crítica do conteúdo do artigo e revisão final do texto.

\section{Agradecimentos}

Este artigo é parte da dissertação de Mestrado do autor principal, e para tanto o apoio financeiro da Coordenação de Aperfeiçoamento de Pessoal de Nível Superior e da Agência Nacional de Vigilância Sanitária, respectivamente no curso do Mestrado e na finalização da dissertação, foram fundamentais. Agradece aos Profs. Paulo Roberto Vasconcellos-Silva, Kenneth Rochel de Camargo Jr. e Luis David Castiel pela contribuição teórico-conceitual durante as fases de elaboração e defesa da dissertação.

\section{Referências}

1. Education for Physician on End-of-Life Care. EPEC Participant's handbook: Plenary 1 - gaps in endof-life care. Chicago: The Robert Wood Johnson Foundation; 1999.

2. World Health Organization. National cancer control programmes: policies and managerial guidelines. 2nd Ed. Geneva: World Health Organization; 2002.

3. Milicevic N. The hospice movement: history and current worldwide situation. Archive of Oncology 2002; 10:29-32.

4. Figueiredo MTA. International Association for Hospice \& Palliative Care News On-line. Regional News Brazil. http://www.hospicecare.com/news letter2004/april04/page5.html (acessado em 03/ Nov/2004).

5. Silva RCF. Cuidados paliativos oncológicos: reflexões sobre uma proposta inovadora na atenção à saúde [Dissertação de Mestrado]. Rio de Janeiro: Escola Nacional de Saúde Pública, Fundação Oswaldo Cruz; 2004.

6. Doyle D, Hanks GWC, Mac Donald N, editors. Oxford textbook of palliative medicine. Oxford: Oxford University Press; 1998.

7. Singer P. Quality end-of-life: a global perspective. BMC Palliat Care 2002; 1:4. http://www.biomedcentral.com/1472-684X/1/4 (acessado em 02/ Set/2003).

8. Higginson IJ. Evidence based palliative medicine. BMJ 1999; 319:462-3.

9. Tolstoi L. A morte de Ivan Ilitch. Porto Alegre: L\&PM Editores; 1997.

10. Fordham S, Dorwick C. Is care of the dying improving? The contribution of specialist and nonspecialist to palliative care. Fam Pract 1999; 16: 573-9.

11. Morrison R, Meier D. Palliative care. N Engl J Med 2004; 350:2582-90.

12. Elsayem A, Swint K, Fisch M, Palmer J, Reddy S, Walker $\mathrm{P}$, et al. Palliative care inpatient service in a comprehensive cancer center: clinical and financial outcomes. J Clin Oncol. 2004; 22:200814

13. National Consensus Project for Quality Palliative Care. Clinical practice guidelines for quality palliative care. Pittsburgh: National Consensus Project for Quality Palliative Care; 2004.

14. Palliative Care Australia. Standards for palliative care provision. 3rd Ed. [s.l.]: Palliative Care Australia; 1999.

15. Schramm FR. Morte e finitude em nossa sociedade: implicações no ensino dos cuidados paliativos. Rev Bras Cancerol 2002; 48:17-20.

16. Da Matta R. A casa e a rua: espaço, cidadania, mulher e morte no Brasil. 5a Ed. Rio de Janeiro: Editora Rocco; 2000.

17. Vianna A, Piccelli H. O estudante, o médico e o professor de medicina perante a morte e o paciente terminal. AMB Rev Assoc Med Bras 1998; 44:21-7.

18. Hennezel M, Leloup J. A arte de morrer: tradições religiosas e espiritualidade humanista diante da morte na atualidade. Petrópolis: Editora Vozes; 1999. 
19. Clark D. Between hope and acceptance: the medicalisation of dying. BMJ 2002; 324:905-7.

20. Boff L. Saber cuidar: ética do humano - compaixão pela terra. 4a Ed. Petrópolis: Editora Vozes, 1999.

21. Caponi S. Da compaixão à solidariedade. Rio de Janeiro: Editora Fiocruz; 2000

22. Ramirez A, Addington-Hall J, Richards M. The carers. BMJ 1998; 324:1291-2.

23. Karsch UM. Idosos dependentes: famílias e cuidadores. Cad Saúde Pública 2003; 19:861-6.
24. Floriani CA, Schramm FR. Atendimento domiciliar ao idoso: problema ou solução. Cad Saúde Pública 2004; 20:986-94.

25. Maslach C, Schaufeli WB, Leiter MP. Job Burnout. Annu Rev Psychol 2001, 52:397-422.

26. Caldas CP. Envelhecimento com dependência: responsabilidades e demandas da família. Cad Saúde Pública 2003; 19:773-81.

Recebido em 14/Jan/2005

Aprovado em 21/Fev/2006 